\title{
Mejoras en la programación de la producción de una empresa farmacéutica
}

Eddy Pacoricuna Cabrera* Miguel Mejía Puente**

\section{RESUMEN}

En una empresa farmacéutica se identificaron las causas del bajo nivel de cumplimiento en el plan de producción, de la constante acumulación de lotes por fabricar y del exceso de horas-hombre durante los procesos de envasado y acondicionado en la línea de líquidos no estériles. Luego de analizar las causas principales de estas deficiencias se propusieron dos mejoras: la primera es una línea automática que se encargaría de realizar las operaciones de dosificado, tapado y etiquetado y la segunda se trata de un modelo matemático que propone un programa de producción de la línea de líquidos no estériles. Las mejoras planteadas permitirán un ahorro anual de S/. 381,925 con un retorno de la inversión de 1,11 años.

Palabras clave: múltiples objetivos, programa de producción, programación lineal entera mixta

IMPROVED PRODUCTION SCHEDULING OF A PHARMACEUTICAL COMPANY

\section{ABSTRACT}

In a pharmaceutical company were identified the causes of low level of compliance in the production plan, of the constant backlog of lots for manufacturing and the excessive hours-man during the packaging and conditioning processes in the line of non-sterile liquids. After analyzing the main causes of these deficiencies two improvements are proposed: The first is an automatic line that would be responsible for performing the operations of dosing, capping and labeling and the second is a mathematical model that offers a program of production for non-sterile liquid line. The proposed improvements will allow annual savings of S/. 385,925 with a return of investment 1,11 years.

Keywords: mixed integer linear programming, multiply objectives, schedule production

\section{INTRODUCCIÓN}

La investigación se realiza en una empresa farmacéutica que se encuentra en una etapa de crecimiento debido a la conformación de la cadena de abastecimiento de productos genéricos y de marca. La competitividad del sector farmacéutico hace necesario que la empresa mejore sus procesos para poder atender la demanda de productos. El estudio consiste en dos propuestas de mejora para la línea de producción de líquidos no estériles. La primera mejora tiene que ver con la adquisición e implementación de una línea de dosificado, tapado y etiquetado automática que reduce los costos de horas-hombre en los proceso de envasado y acondicionado, y la segunda mejora se basa en el desarrollo de un modelo matemático que permite obtener un programa de producción para reducir los costos productivos y para incrementar el nivel de atención de demanda.

\section{CASO DE ESTUDIO}

La empresa farmacéutica a estudiar se ubica en la ciudad de Lima. Esta cuenta con cuatro unidades de negocio que son la unidad productiva con maquinaria especializada en la fabricación, envasado y acondicionado de productos farmacéuticos; la unidad distribuidora encargada de almacenar y distribuir productos farmacéuticos; la unidad comercializadora encargada de posicionar los productos en farmacias, boticas, centros hospitalarios y clínicas; y la unidad de mercadeo conformada por una cadena de boticas ubicadas en todo el territorio nacional. Esta empresa ha construido una cadena de valor sanitaria integrada por producción, distribución, comercialización y mercadeo de productos farmacéuticos.

\section{Sistema de producción}

A continuación se describirán brevemente los productos, la tipología del sistema productivo y la descripción de los procesos principales desde la planificación hasta la obtención del producto terminado.

Los productos pertenecen a cuatro grupos o líneas de producción: sólidos (tabletas, cápsulas, grageas y polvos), líquidos no

\footnotetext{
Ingeniero Industrial, PUCP. Analista Comercial, Motocorp S.A.C

E-mail: eddy.pacoricuna@motocorp.pe

* Doctor en Ingeniería Industrial. Docente principal del Departamento de Ingeniería, PUCP.

E-mail: miguel.mejia@pucp.edu.pe
} 
estériles (jarabes y suspensiones), líquidos estériles (inyectables y oftálmicos) y semisólidos (cremas, supositorios y óvulos).

El estudio se enfocará en la línea de los productos líquidos no estériles. Estos son 40 productos, de los cuales 31 son jarabes y 9 son suspensiones.

La tipología del sistema de producción corresponde a una producción por lotes con cuatro características principales. En primer lugar, el inicio de la fabricación es posible solo si se tiene la Orden de Trabajo, es decir, existe un pedido que solicita el producto para despacharlo al cliente. En segundo lugar, se cuenta con tres clientes denominados A, B y C. En tercer lugar, la variedad de productos está conformada por 40 diferentes productos; y por último, la demanda en el sector farmacéutico es fluctuante, lo que hace difícil obtener un pronóstico de fabricación en un periodo de tiempo mayor a 6 meses.

El sistema de producción actual se puede resumir en el Diagrama de Flujo de Datos (DFD) que se presenta en el Gráfico 1. Aquí se muestra el flujo de datos e informaciones que se obtiene en el proceso de planeamiento, logística y despacho.

Como se puede apreciar en el DFD del proceso principal, las áreas involucradas son planeamiento, marketing, logística, producción, almacenes, despacho y contabilidad. El estudio se centrará en las áreas de planeamiento y producción que corresponden a los procesos Planear y Producir.
El análisis de la situación actual de los procesos 1 y 3 permitió identificar lo siguiente:

- El nivel de cumplimiento del plan depende de una correcta programación de la producción y un adecuado proceso de envasado. Hasta el momento solo se ha cumplido con el $64 \%$ del plan programado por lo que se está dejando de percibir ingresos en la línea de productos no estériles.

- El plan de producción solo se puede ejecutar si se dispone de los insumos necesarios (materia prima y envases), por ello también se debe planificar el requerimiento de estos.

- El plan que se ha estado manejando no contempla los trabajos preventivos de mantenimiento, por ello los jefes de producción no tienen disponibilidad diaria en la fabricación.

- Actualmente la política de secuenciación está basada en las urgencias de entrega de los productos que se establecen con los clientes. Este hecho hace que se pierdan oportunidad de optimizar los procesos adecuadamente.

- El proceso de envasado es muy lento y requiere la disponibilidad de varios operarios. Logrando automatizar las operaciones se podría disponer de estos operarios a otras líneas de producción que lo necesitan.

Gráfico 1. Diagrama de flujo de datos del proceso principal.

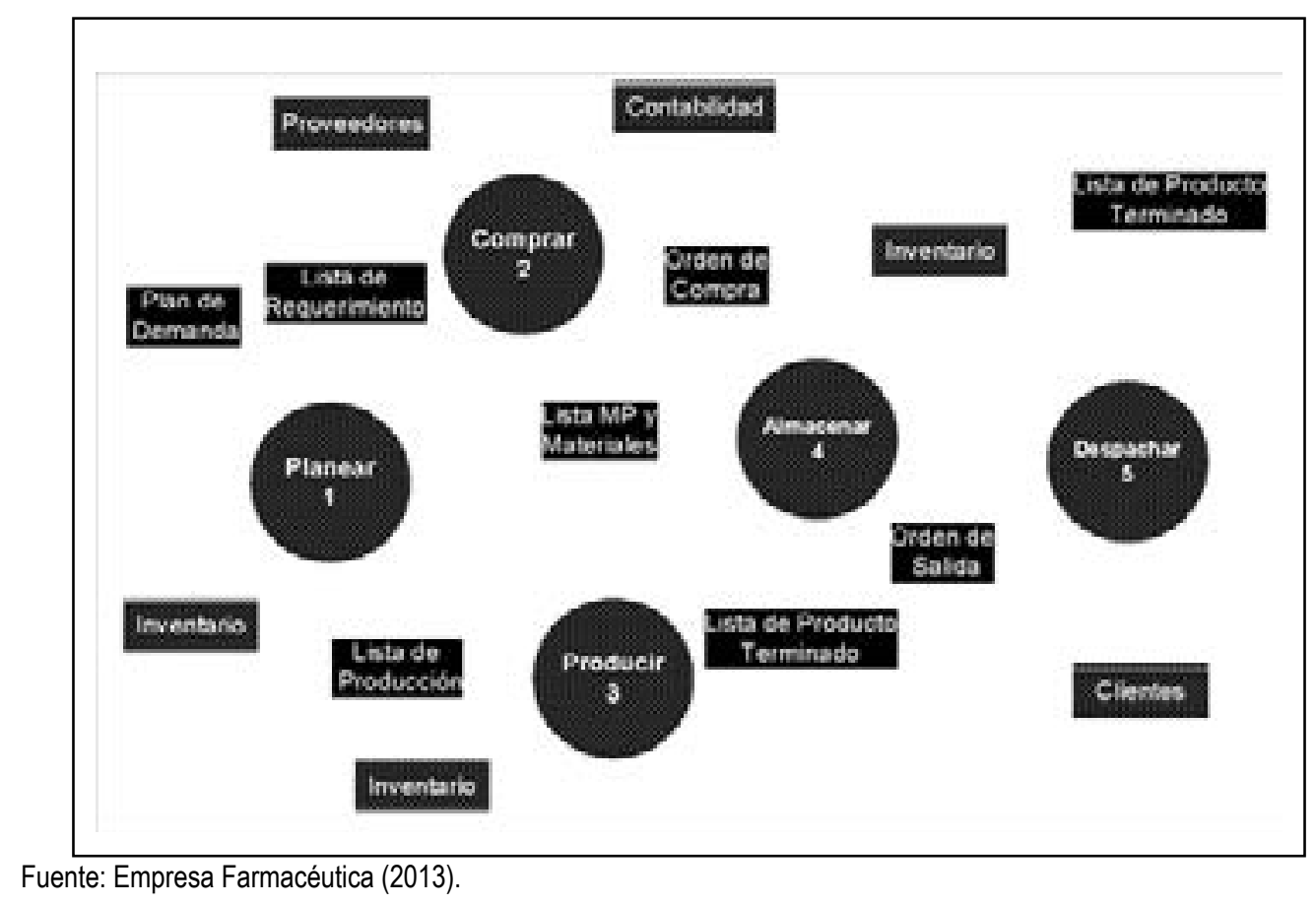




\section{Descripción de los problemas}

Para encontrar los principales problemas se utilizaron herramientas de "lluvias de ideas" y análisis causa-efecto. Con la "lluvia de ideas" se identificaron los siguientes problemas:

- Se pierde oportunidad de obtener más ingresos.

- Hay una falta de optimización en el proceso de fabricación.

- El proceso de envasado restringe el uso de tanques.

Luego de aplicar el análisis causa-efecto a cada uno de los tres problemas anteriores, se identificaron las siguientes propuestas de mejora:

- Obtener un programa de producción que optimice la secuencia considerando los turnos extras (sábados y domingos) y los insumos disponibles.

- Renovar los equipos de envasado para que el proceso sea más eficiente en todos los productos.

\section{PLAN DE MEJORAS}

Primero, se realizará una mejora en el proceso de envasado mediante la implementación de una línea automática que reduzca el tiempo de espera del proceso en menos de un día por cada lote de producto. Luego, con la primera mejora implementada, se elaborará un programa de producción mensual usando un modelo de programación lineal entera mixta por metas, el cual nos ayudará a encontrar una secuencia óptima de producción tal que podamos maximizar las utilidades y mejorar la utilización de los recursos disponibles.

\section{Línea automática de dosificado, tapado y etiquetado}

Para llevar a cabo la primera mejora, se escogió el producto llamado suspensión 37 como el más representativo por su alta demanda.

Para lograr una reducción significativa de tiempo se sugirió la adquisición de una línea automática del proveedor VC Seraming S.R.L. Esta línea realiza las operaciones principales de dosificado, tapado y etiquetado. Con el nuevo equipo solo se necesita cuatro operarios que se encarguen del monitoreo y control en las tres operaciones que realiza. Según las especificaciones dadas por el proveedor, esta línea es capaz de procesar 5,760 unidades en una hora. Es decir, puede llenar, tapar y etiquetar un lote del producto suspensión 37 en una hora.

\section{Modelación del programa de producción}

El plan proyectado que actualmente se aplica en el área de planeamiento está realizado según la política de stocks por coberturas que implica fabricar los productos con un bajo nivel de stock. Por este motivo, el plan proyectado no permite optimizar los procesos de producción en la línea, y tampoco permite ahorrar en costos extras que se incurren para cumplir con las urgencias.

Para mitigar este inconveniente se plantea realizar un modelo de programación lineal entera mixta por metas que nos indique cuántos lotes de cada producto fabricar y en qué días se deben realizar para atender la demanda en el plan proyectado considerando la capacidad de la planta; los insumos disponibles en el almacén y la prioridad de atención a los clientes tal que se puedan maximizar las utilidades con un impacto positivo en el nivel de cumplimiento del plan proyectado.

\section{Supuestos del modelo}

- El periodo de estudio del modelo es de cinco meses los cuales comprende de enero a mayo de 2013. Cada mes cuenta con un determinado número de días operativos divididos en días de turno normal y turno extra.

- El inventario de insumos disponibles (materia prima y envases) es dato de entrada y se verifica en el sistema de stock de inventarios con el que cuenta la empresa, antes del inicio de cada mes.

- La materia prima que se considera en el modelo es el principio activo, ya que es el principal componente de cada producto.

- La demanda de productos se encuentra en el plan de producción y es dato de entrada para la programación. Los productos que no se fabriquen se acumulan para el siguiente periodo.

- El mezclado, filtrado, envasado y acondicionado se realizan de manera consecutiva y de acuerdo al tiempo de entrega en cada proceso.

- Los costos de materia prima, mano de obra directa (operarios) e indirecta (supervisores) se considerarán como costos variables que solo se incurren si se toma la decisión de procesar un lote de producto. El ahorro considerado en la operación de mezclado de suspensión debido a la producción en campaña fueron calculados mediante el juicio de los operarios y supervisores considerando a la mano de obra como la principal fuente de ahorro.

- El orden de prioridad de atención a los clientes se basa en la cobertura determinada para cada 
uno. El cliente que requiere la mayor cobertura en sus productos es aquel que tiene la mayor prioridad.

\section{Formulación del modelo}

\section{Parámetros}

$t d_{m}$ : Cantidad total de días con el que cuenta el mes $\mathrm{m}$

$P v j a b_{i}$ : Precio de venta del jarabe i

Pvsus $_{j}$ : Precio de venta de la suspensión j

$\operatorname{Cpm}_{d} J_{i}$ : Costo de mezclar en el día d un lote del jarabe i

$\operatorname{Cpm}_{d} S_{1 j}$ : Costo de mezclar en el día d un lote de la suspensión j

$\operatorname{Cpm}_{d} S_{2 j}$ : Costo de mezclar en el día d un lote campaña de la suspensión j

$\operatorname{Cpf}_{d} J_{i}$ : Costo de filtrar en el día d un lote del jarabe i

$\operatorname{Cpf}_{d} S_{1 j}$ : Costo de filtrar en el día d un lote de la suspensión j

$\operatorname{Cpf}_{d} S_{2 j}$ : Costo de filtrar en el día d un lote campaña de la suspensión j

$\operatorname{Cpe}_{d} J_{i}$ : Costo de envasar en el día d un lote del jarabe $\mathrm{i}$

$C p e_{d} S_{1 j}$ : Costo de envasar en el día d un lote de la suspensión j

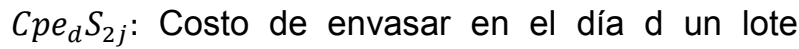
campaña de la suspensión j

$C p a_{d} J_{i}$ : Costo de acondicionar en el día d un lote del jarabe i

$C p a_{d} S_{1 j}$ : Costo de acondicionar en el día d un lote de la suspensión j

$C p a_{d} S_{2 j}$ : Costo de acondicionar en el día d un lote campaña de la suspensión j

dia $a_{d}$ : 1 si el área de fabricación está disponible para trabajar en el día d, 0 en caso contrario

$J a b D_{i}$ : Cantidad de lotes del jarabe i que se demandan en el mes
$S u s D_{j}$ : Cantidad de lotes de la suspensión j que se demandan en el mes

$M P o_{w}$ : Cantidad de kilogramos de materia prima w que existen al inicio del mes

$v c m j a b_{i}$ : Factor de conversión de lote a materia prima para un lote del jarabe i

vcmsus $_{j}$ : Factor de conversión de lote a materia prima para un lote de suspensión j

$E \mathrm{No}_{\mathrm{z}}$ : Cantidad de unidades de envases z que existe al inicio del mes

$v c e j a b_{i}$ : Factor de conversión de lote a envases para un lote del jarabe i

vcesus $_{j}$ : Factor de conversión de lote a envases para un lote de la suspensión j

\section{Variables de decisión}

$M_{d} J_{i}$ : Decisión de mezclar en el día d un lote del jarabe i

$M_{d} S_{1 j}$ : Decisión de mezclar en el día d un lote de la suspensión j

$M_{d} S_{2 j}$ : Decisión de mezclar en el día d un lote campaña de la suspensión j

$F_{d} J_{i}$ : Decisión de filtrar en el día d un lote del jarabe i

$F_{d} S_{1 j}$ : Decisión de filtrar en el día d un lote de la suspensión j

$F_{d} S_{2 j}$ : Decisión de filtrar en el día d un lote campaña de la suspensión j

$E_{d} J_{i}$ : Decisión de envasar en el día d un lote del jarabe i

$E_{d} S_{1 j}$ : Decisión de envasar en el día d un lote de la suspensión j

$E_{d} S_{2 j}$ : Decisión de envasar en el día d un lote campaña de la suspensión j

$A_{d} J_{i}$ : Decisión de acondicionar en el día d un lote del jarabe i

$A_{d} S_{1 j}$ : Decisión de acondicionar en el día d un lote de la suspensión j 
$A_{d} S_{2 j}$ : Decisión de acondicionar en el día d un lote campaña de la suspensión j

$J a b P_{i}$ : Cantidad de lotes del jarabe $\mathrm{i}$ que se producen en el mes

$J a b N P_{i}$ : Cantidad de lotes del jarabe i que se dejan de producir en el mes

$\operatorname{Sus}_{j}$ : Cantidad de lotes de la suspensión j que se producen en el mes

SusN $P_{j}$ : Cantidad de lotes de la suspensión j que se dejan de producir en el mes

$M P U_{w}$ : Cantidad de kilogramos de materia prima w utilizados en la producción del mes.

$M P f_{w}$ : Cantidad de kilogramos de materia prima w que queda al final del mes

$E N U_{z}$ : Cantidad de unidades de envases z utilizados en la producción del mes

$E N f_{z}$ : Cantidad de unidades de envases z que quedan al final del mes

\section{Función objetivo}

Minimizar $Z=P_{1} * U_{1}+P_{2} * U_{2}+P_{3} * U_{3}$

Los objetivos por orden de prioridad son los siguientes:

1. Maximizar la utilidad $\sin$ costos fijos considerando únicamente los productos del cliente 1

2. Maximizar la utilidad sin costos fijos considerando únicamente los productos del cliente 2

3. Maximizar la utilidad sin costos fijos considerando únicamente los productos del cliente 3

4.

\section{Restricciones de metas}

Maximizar la utilidad sin costos fijos considerando únicamente los productos del cliente 1

$\left(\right.$ Pvjab $\left._{i} * \operatorname{JabP}_{i}\right)+\left(\right.$ Pvsus $\left._{j} * \operatorname{SusP}_{j}\right)-$

$\sum_{d=1}^{t d_{m}} \quad\left(C p m_{d} J_{i} * M_{d} J_{i}\right)-\sum_{d=1}^{t d_{m}-1} \quad\left(C p m_{d} S_{1 j} *\right.$

$\left.M_{d} S_{1 j}\right)$ $-\sum_{d=1}^{t d_{m}-2} \quad\left(C p m_{d} S_{2 j} * M_{d} S_{2 j}\right)-\sum_{d=1}^{t d_{m}} \quad\left(C p f_{d} J_{i} *\right.$

$\left.F_{d} J_{i}\right)-\sum_{d=2}^{t d_{m}} \quad\left(C p f_{d} S_{1 j} * F_{d} S_{1 j}\right)$

$-\sum_{d=3}^{t d_{m}} \quad\left(C p f_{d} S_{2 j} * F_{d} S_{2 j}\right)-\sum_{d=2}^{t d_{m}+1} \quad\left(C p e_{d} J_{i} *\right.$

$\left.E_{d} J_{i}\right)-\sum_{d=3}^{t d_{m}+1}\left(C_{p e} S_{1 j} * E_{d} S_{1 j}\right)$

$-\sum_{d=4}^{t d_{m}+1} \quad\left(C p e_{d} S_{2 j} * E_{d} S_{2 j}\right)-\sum_{d=3}^{t d_{m}+2} \quad\left(C p a_{d} J_{i} *\right.$

$\left.A_{d} J_{i}\right)-\sum_{d=4}^{t d_{m}+2}\left(C^{2} a_{d} S_{1 j} * A_{d} S_{1 j}\right)$

$-\sum_{d=5}^{t d_{m}+2}\left(C p a_{d} S_{2 j} * A_{d} S_{2 j}\right)+U_{1}-V_{1}=$

$M ; \forall i=7, \ldots 12,24, \ldots 31 ; \forall j=1, \ldots 4$.

Maximizar la utilidad sin costos fijos considerando únicamente los productos del cliente 2

$\left(\right.$ Pvjab $\left._{i} * \operatorname{JabP}_{i}\right)+\left(\right.$ Pvsus $_{j} *$ SusP $\left._{j}\right)-$

$\sum_{d=1}^{t d_{m}} \quad\left(C_{p} m_{d} J_{i} * M_{d} J_{i}\right)-\sum_{d=1}^{t d_{m}-1} \quad\left(C p m_{d} S_{1 j} *\right.$

$\left.M_{d} S_{1 j}\right)$

$-\sum_{d=1}^{t d_{m}-2} \quad\left(C p m_{d} S_{2 j} * M_{d} S_{2 j}\right)-\sum_{d=1}^{t d_{m}} \quad\left(C p f_{d} J_{i} *\right.$

$\left.F_{d} J_{i}\right)-\sum_{d=2}^{t d_{m}} \quad\left(C p f_{d} S_{1 j} * F_{d} S_{1 j}\right)$

$-\sum_{d=3}^{t d_{m}} \quad\left(C p f_{d} S_{2 j} * F_{d} S_{2 j}\right)-\sum_{d=2}^{t d_{m}+1} \quad\left(C_{p e} J_{d} *\right.$

$\left.E_{d} J_{i}\right)-\sum_{d=3}^{t d_{m}+1}\left(\mathrm{Cpe}_{d} S_{1 j} * E_{d} S_{1 j}\right)$

$-\sum_{d=4}^{t d_{m}+1}\left(C p e_{d} S_{2 j} * E_{d} S_{2 j}\right)-\sum_{d=3}^{t d_{m}+2} \quad\left(C p a_{d} J_{i} *\right.$

$\left.A_{d} J_{i}\right)-\sum_{d=4}^{t d_{m}+2} \quad\left(C p a_{d} S_{1 j} * A_{d} S_{1 j}\right)$

$-\sum_{d=5}^{t d_{m}+2} \quad\left(C p a_{d} S_{2 j} * A_{d} S_{2 j}\right)+U_{2}-V_{2}=$ $M ; \forall i=1, \ldots 4,13, \ldots 22 ; \forall j=5, \ldots 9$.

Maximizar la utilidad sin costos fijos considerando únicamente los productos del cliente 3

$\left(\right.$ Pvjab $\left._{i} * J a b P_{i}\right)-\sum_{d=1}^{t d_{m}} \quad\left(C_{d p m_{d} J_{i}} * M_{d} J_{i}\right)-$ $\sum_{d=1}^{t d_{m}} \quad\left(C p f_{d} J_{i} * F_{d} J_{i}\right)-\sum_{d=2}^{t d_{m}+1} \quad\left(C p e_{d} J_{i} * E_{d} J_{i}\right)$

$-\sum_{d=3}^{t d_{m}+2}\left(C p a_{d} J_{i} * A_{d} J_{i}\right)+U_{3}-V_{3}=M ; \forall i=$ $5,6,23$.

\section{Restricciones estructurales}

\section{Planificación}

Atención de lotes demandados de productos de la forma jarabes

$J a b P_{i}+J a b N P_{i}=J a b D_{i} ; \forall i,=1, \ldots 31$ 
Atención de lotes demandados de productos de la forma suspensiones

$\operatorname{Sus} P_{j}+\operatorname{SusN}_{j}=\operatorname{SusD}_{j} ; \forall j=1, \ldots 9$

Disponibilidad de materia prima

$M P U_{w}+M P f_{w}=M P o_{w} ; \forall w=1, \ldots 25$

Disponibilidad de envases

$E N U_{z}+E N f_{z}=E N o_{z} ; \forall z=1, \ldots, 26$

\section{Línea de fabricación del producto}

Capacidad del proceso de mezclado sin lote campaña

$\sum_{i=1}^{31} \quad M_{d} J_{i}+\sum_{j=1}^{9} \quad M_{d} S_{1 j} \leq d i a_{d} ; \forall d=$

$1, \ldots t d_{m}-1$

$\sum_{i=1}^{31} \quad M_{d} J_{i} \leq d i a_{d} ; \forall d=t d_{m}$

Capacidad del proceso de mezclado con lote campaña

$\sum_{j=1}^{9} \quad M_{d} S_{2 j} \leq d i a_{d} ; \forall d=1, \ldots t d_{m}-2$

Capacidad del proceso de filtrado sin lote campaña

$\sum_{i=1}^{31} \quad F_{d} J_{i} \leq d i a_{d} ; \forall d=1$

$\sum_{i=1}^{31} \quad F_{d} J_{i}+\sum_{j=1}^{9} \quad F_{d} S_{1 j} \leq d i a_{d} ; \forall d=2$

Capacidad del proceso de filtrado con lote campaña

$\sum_{i=1}^{31} \quad F_{d} J_{i}+\sum_{j=1}^{9} \quad F_{d} S_{1 j}+\sum_{j=1}^{9} \quad F_{d} S_{2 j} \leq$ $\operatorname{dia}_{d} ; \forall d=3, \ldots t d_{m}$

Relación entre el mezclado de dos lotes que se producen en campaña

$M_{d} S_{2 j}-M_{d} S_{1 j} \leq 0 ; \forall d=1, \ldots t d_{m}-2 . \forall j=$ $1, \ldots, 9$

Relación entre el filtrado de dos lotes que se producen en campaña

$F_{(d+1)} S_{2 j}-F_{d} S_{1 j} \leq 0 ; \forall d=2, \ldots, t d_{m}-1 ; \quad \forall j=$ $1, \ldots 9$

Relación entre el mezclado y el filtrado de cualquier lote de una suspensión $\sum_{j=1}^{9} \quad F_{d} S_{1 j}+\sum_{j=1}^{9} \quad M_{d} S_{1 j} \leq 1 ; \forall d=2$ $\sum_{j=1}^{9} \quad F_{d} S_{1 j}+\sum_{j=1}^{9} \quad F_{d} S_{2 j}+\sum_{j=1}^{9} \quad M_{d} S_{1 j} \leq$

$1 ; \forall d=3, \ldots t d_{m}-1$

Relación entre el proceso de mezclado y filtrado de jarabes

$F_{d} J_{i}-M_{d} J_{i} \leq 0 ; \forall i=1, \ldots 31 ; \forall d=1, \ldots t d_{m}$

$M_{d} J_{i}-F_{d} J_{i} \leq 0 ; \forall i=1, \ldots 31 ; \forall d=1, \ldots t d_{m}$

Relación entre el proceso de mezclado y filtrado de suspensiones sin lotes campaña

$F_{(d+1)} S_{1 j}-M_{d} S_{1 j} \leq 0 ; \forall j=1, \ldots 9 ; \forall d=$

$1, \ldots t d_{m}-1$

$M_{d} S_{1 j}-F_{(d+1)} S_{1 j} \leq 0 ; \forall j=1, \ldots 9 ; \forall d=$

$1, \ldots t d_{m}-1$

Relación entre el proceso de mezclado y filtrado de suspensiones de lotes campaña

$F_{(d+2)} S_{2 j}-M_{d} S_{2 j} \leq 0 ; \forall j=1, \ldots 9 ; \forall d=$

$1, \ldots t d_{m}-2$

$M_{d} S_{2 j}-F_{(d+2)} S_{2 j} \leq 0 ; \forall j=1, \ldots 9 ; \forall d=$

$1, \ldots t d_{m}-2$

Política actual para el inicio de fabricación de los productos controlados

$M_{d} J_{i}=0 ; \forall d=1, \ldots 19 ; \forall i=3,7,8,18,21,22$

Balance entre materia prima y los productos realizados

$M P U_{w}=$ $v_{c m j a b_{i}} * \operatorname{JabP}_{i}+$ vcmsus $_{j} * \operatorname{Sus}_{j} ; \forall \boldsymbol{w}=$

$1, \ldots 25 ; \forall i=1, \ldots 31 ; \forall j=1, \ldots 9$

Relación entre los jarabes producidos y el proceso de mezcla

$J a b P_{i}-\sum_{d=1}^{30} \quad M_{d} J_{i}=0 ; \forall i=1, \ldots 31$

Relación entre las suspensiones producidas y el proceso de mezcla

$\operatorname{SusP}_{j}-\sum_{d=1}^{t d_{m}-1} \quad M_{d} S_{1 j}-\sum_{d=1}^{t d_{m}-2} \quad M_{d} S_{2 j}=$ $0 ; \forall j=1, \ldots 9$ 


\section{Línea de envasado del producto}

Capacidad del proceso de envasado sin lote campaña

$\sum_{i=1}^{31} \quad E_{d} J_{i} \leq d i a_{d} ; \forall d=2$

$\sum_{i=1}^{31} \quad E_{d} J_{i}+\sum_{j=1}^{9} \quad E_{d} S_{1 j} \leq d i a_{d} ; \forall d=3$

Capacidad del proceso de envasado con lote campaña

$\sum_{i=1}^{31} \quad E_{d} J_{i}+\sum_{j=1}^{9} \quad E_{d} S_{1 j}+\sum_{j=1}^{9} \quad E_{d} S_{2 j} \leq$

$d i a_{d} ; \forall d=4, \ldots t d_{m}+1$

Relación entre el proceso de filtrado y envasado de un lote de un jarabe

$E_{(d+1)} J_{i}-F_{d} J_{i} \leq 0 ; \forall i=1, \ldots, 31 ; \forall d=1, \ldots t d_{m}$ $F_{d} J_{i}-E_{(d+1)} J_{i} \leq 0 ; \forall i=1, \ldots, 31 ; \forall d=1, \ldots t d_{m}$

Relación entre el proceso de filtrado y envasado de un lote de una suspensión

$E_{(d+1)} S_{1 j}-F_{d} S_{1 j} \leq 0 ; \forall j=1, \ldots, 9 ; \forall d=2, \ldots t d_{m}$ $F_{d} S_{1 j}-E_{(d+1)} S_{1 j} \leq 0 ; \forall j=1, \ldots, 9 ; \forall d=$ $2, \ldots t d_{m}$

Relación entre el proceso de filtrado y envasado de un lote campaña de suspensión

$E_{(d+1)} S_{2 j}-F_{d} S_{2 j} \leq 0 ; \forall j=1, \ldots, 9 ; \forall d=3, \ldots t d_{m}$ $F_{d} S_{2 j}-E_{(d+1)} S_{2 j} \leq 0 ; \forall j=1, \ldots 9 ; \forall d=3, \ldots t d_{m}$

Restricción de lotes grandes mayor a 10,000 unidades

$$
\begin{gathered}
E_{d} J_{3}+E_{d} J_{7}+E_{d} J_{8}+E_{d} J_{18}+E_{d} J_{21}+E_{d} J_{22}+E_{d+1} J_{3} \\
\quad+E_{d+1} J_{7}+E_{d+1} J_{8} \\
+E_{d+1} J_{18}+E_{d+1} J_{21}+E_{d+1} J_{22} \leq 0 ; \forall d=2, \ldots t d_{m}
\end{gathered}
$$

Balance entre los envases y los productos $E N U_{z}=\operatorname{vcejab}_{i} * \operatorname{JabP}_{i}+$ vcesus $_{j} * \operatorname{Sus}_{j} ; \quad \forall w=$ $1, \ldots, 25 . \forall i=1, \ldots, 31 . \forall j=1, \ldots, 9$

\section{Línea de acondicionado del producto}

Capacidad del proceso de acondicionado sin lotes campaña

$\sum_{i=1}^{31} \quad A_{d} J_{i} \leq 1 ; \forall d=3$

$\sum_{i=1}^{31} \quad A_{d} J_{i}+\sum_{j=1}^{9} \quad A_{d} S_{1 j} \leq 1 ; \forall d=4$
Capacidad del proceso de acondicionado con lotes campaña

$\sum_{i=1}^{31} \quad A_{d} J_{i}+\sum_{j=1}^{9} \quad A_{d} S_{1 j}+\sum_{j=1}^{9} \quad A_{d} S_{2 j} \leq$

$1 ; \forall d=5, \ldots t d_{m}+2$

Relación entre el proceso de envasado y acondicionado de un lote de un jarabe

$A_{(d+1)} J_{i}-E_{d} J_{i} \leq 0 ; \forall i=1, \ldots, 31 ; \forall d=$

$2, \ldots t d_{m}+1$

$E_{d} J_{i}-A_{(d+1)} J_{i} \leq 0 ; \forall i=1, \ldots, 31 ; \forall d=$

$2, \ldots t d_{m}+1$

Relación entre el proceso de envasado y acondicionado de un lote de una suspensión

$A_{(d+1)} S_{1 j}-E_{d} S_{1 j} \leq 0 ; \forall j=1, \ldots 9 ; \forall d=$

$3, \ldots t d_{m}+1$

$E_{d} S_{1 j}-A_{(d+1)} S_{1 j} \leq 0 ; \forall j=1, \ldots 9 ; \forall d=$

$3, \ldots t d_{m}+1$

Relación entre el proceso de envasado y acondicionado de un lote campaña de una suspensión

$A_{(d+1)} S_{2 j}-E_{d} S_{2 j} \leq 0 ; \forall j=1, \ldots 9 ; \quad \forall d=$

$4, \ldots t d_{m}+1$

$E_{d} S_{2 j}-A_{(d+1)} S_{2 j} \leq 0 ; \forall j=1, \ldots 9 ; \forall d$

$$
=4, \ldots t d_{m}+1
$$

\section{ANÁLISIS DE RESULTADOS}

Se realizó la modelación para los meses de enero hasta mayo del 2013 obteniéndose una utilidad total de S/.73,081.00 por encima de los resultados reales de la empresa en el mismo periodo de producción y, además, se logró producir 15 lotes más que la empresa. Los resultados se muestran en la Tabla 1.

El incremento en la producción de lotes ha tenido un efecto positivo en el nivel de atención de demanda. Según las propuestas del modelo para los meses de estudio, el nivel de atención ha tenido un incremento de 64 a $78 \%$. En la Tabla 2 se aprecia el nivel de atención obtenido en cada mes de producción. 


\section{Resumen del análisis económico}

El análisis de costo-beneficio se muestra en la Tabla 3.

Se demuestra que desde el punto de vista financiero, las mejoras implementadas tienen capacidad de generación valor para la empresa. En total se obtendría un beneficio anual de con un retorno a la inversión de 1.11.

\section{CONCLUSIONES}

Las principales conclusiones son:

- La línea de automática genera un ahorro de S/.229 416 al año y también aumenta la capacidad disponible con lo cual se procesarían más unidades de producción al mes.

- El programa de producción planteado por el modelo matemático nos permite obtener una utilidad de S/.152 509 respecto del programa establecido por la empresa, lo que aumentaría el nivel de atención de demanda a un $78 \%$.

- Las mejoras planteadas permiten un ahorro anual de S/.381 925 con un retorno de la inversión de 1,11 años, lo cual se considera como un proyecto rentable que debe implementarse en el mediano plazo.

Tabla 1. Comparación de resultados

\begin{tabular}{lllll}
\hline \multicolumn{1}{c}{ Año 2013 } & \multicolumn{2}{c}{ Resultados de la empresa } & \multicolumn{2}{c}{ Resultados del modelo } \\
\hline \multicolumn{1}{c}{ Tipo de cliente } & Lotes Producidos & Utilidad Obtenida & Lotes Producidos & Utilidad Obtenida \\
\hline Enero & 16 & $\mathrm{~S} / .48,063$ & 20 & $\mathrm{~S} / .63,123$ \\
Febrero & 4 & $\mathrm{~S} / .12,143$ & 6 & $\mathrm{~S} / .19,554$ \\
Marzo & 14 & $\mathrm{~S} / 34,341$ & 20 & $\mathrm{~S} / .68,172$ \\
Abril & 21 & $\mathrm{~S} / .63,901$ & 21 & $\mathrm{~S} / .66,613$ \\
Mayo & 14 & $\mathrm{~S} / .37,621$ & 17 & $\mathrm{~S} / .51,688$ \\
Total & 69 & $\mathrm{~S} / .196,069$ & 84 & $\mathrm{~S} / .269,150$ \\
\hline
\end{tabular}

Tabla 2. Nivel de atención obtenido por el modelo

\begin{tabular}{lllllll}
\hline \multicolumn{1}{c}{ Periodo } & Ene-13 & Feb-13 & Mar-13 & Abr-13 & May-13 & Total \\
\hline Lotes Planificados & 28 & 20 & 21 & 21 & 18 & 108 \\
Lotes Producidos & 20 & 6 & 20 & 21 & 17 & 84 \\
\%Cumplimiento & $71 \%$ & $30 \%$ & $95 \%$ & $100 \%$ & $94 \%$ & $78 \%$ \\
\hline
\end{tabular}

Tabla 3. Análisis costo-beneficio de las mejoras

\begin{tabular}{lclll}
\hline \multicolumn{1}{c}{ Mejora } & Costo & Beneficio & ROI \\
\hline Línea automática & $\mathrm{S} / .375,740$ & $\mathrm{~S} / .229,416$ & 1.64 & \\
Modelo matemático & $\mathrm{S} / .49,053$ & $\mathrm{~S} / .152,509$ & 0.32 & \\
\hline Total & $\mathrm{S} / .424,793$ & $\mathrm{~S} / .381,925$ & 1.11 & \\
\hline
\end{tabular}




\section{REFERENCIAS BIBLIOGRÁFICAS}

[1] Cuadros, D. (2006). Desarrollo de un modelo de optimización de los procesos productivos de un laboratorio farmacéutico aplicando programación lineal entera mixta con múltiples objetivos. Tesis de Título de Ingeniería Industrial. Pontificia Universidad Católica del Perú. Perú.

[2] Domínguez, J. (1995). Dirección de operaciones: aspectos estratégicos en la producción y los servicios. 1ra. ed. McGrawHill. España.

[3] Mayo, D. (2005). Desarrollo de un modelo de optimización de la producción en una empresa textil utilizando la programación lineal entera mixta. Tesis de Título de Ingeniería Industrial. Pontificia Universidad Católica del Perú. Perú.

[4] Miranda, A. (2010). Análisis y propuesta del sistema de producción de una empresa manufacturera de productos químicos con el enfoque MRP. Tesis de Título de Ingeniería Industrial. Pontificia Universidad Católica del Perú. Perú.

[5] Pacoricuna, E. (2013). Propuesta del programa de producción de la línea de productos líquidos no estériles. Tesis de Título de Ingeniería Industrial. Pontificia Universidad Católica del Perú. Perú. 